\title{
RESISTANCE AND THE TYPING OF LEPROSY SKIN LESIONS
}

\author{
By E. Muir.
}

In recent tours through Africa I found in most of the leprosy institutions visited difficulty and confusion existed in distinguishing the various types of skin lesions and in classifying cases. Yet this classification is most important for prognosis, treatment and control.

Much has been written on this subject, but it is a difficult subject to make lucid. The object of this note is to give a short and simple guide to the recognition of the various types of lesions and their significance.

\section{Resistance.}

The variation in the types of leprosy and in the nature of the lesions may depend to a certain extent on the intensity, duration and nature of the infection, and on the duration of the disease. But the chief factor is not in the seed or in the sowing, but in the soil; it is the resistance or immunity of the patient that is mainly responsible for the nature of the lesion.

Immunity to leprosy may be divided under three main groups: natural, general and acquired.

I. Natural Immminty.-There seems strong evidence that the great majority of people are born with strong natural resistance to leprosy. If infected, they generally either fail to develop any recognised lesion or such lesions as do develop are abortive; or, if progressive disease does occur, it is of the milder neural type.

A small minority of people, perhaps one out of ten, seem to be born with low natural resistance. Even a slight contact may be sufficient to produce the disease, and it tends to be of the more severe lepromatous type.

The evidence that this difference in resistance exists at birth depends on observations such as the following. Two members of one family have been subjected to equal chances of infection; the one acquires the severe lepromatous form of the disease, while the other who is seemingly in poorer general health either escapes altogether or develops only a mild neural lesion. The lepromin test, which is one of the best indexes of resistance to leprosy, shows differences in resistance among children irrespective of their general health.

There is as yet little evidence as to what extent natural resistance varies in its distribution in families or in races. This is a 
matter in urgent need of investigation with the aid of the lepromin tesi.

2. General Kesistance.-As in other diseases, the general health of the patient plays an important part in determining the type of leprosy, the progress of the disease and the nature of the lesion. This factor is the more important because of the long duration of leprosy, during which there is abundant time for complicating conditions to affect the health, and because leprosy itself lowers the health only to a comparatively slight extent.

The chief things which affect the general resistance are complicating diseases, malnutrition, lack of physical exercise, climate and the mental condition. Age also seems to be of importance, young children being less resistant than adults.

Under this heading should also be mentioned localised lowered resistance. Leprous lesions frequently originate in areas of skin, the resistance of which has been impaired by previous injuries such as scars of scabies and septic infections. Also skin lesions tend to be severer and to develop nodules in parts exposed to injury, such as the face and hands.

3. Acquired Resistance.-This may be systemic or local. Even in the most severe lepromatous cases the disease frequently becomes spontaneously eliminated and the infection may entirely die out, leaving only residual deformities. This is apparently the result of acquired immunity.

In tuberculoid skin lesions of the neural type it is common to find the disease spreading at the margin and simultaneously dying out in the centre. This central healing is obviously the result of local acquired immunity.

\section{Types of Leprosy.}

Leprosy is divided into lepromatous and neural types. The former is associated with high, and the latter with low, resistance. While the degree of natural immunity may be the main determinant of the type of disease, the general resistance has an important bearing on its extent and progress. Between the naturally susceptible and the naturally immune patient, there appears to be a threshold, as is shown by the clear cut differences between the nature of the skin lesions found in each. Thus the typically lepromatous lesion is very distinct from the typically tuberculoid lesion found in the neural type. This threshold is probably higher in some communities than in others.

Before discussing further the significance of leprous skin lesions it will be well to enumerate them in tabular form, placing the most typically lepromatous to the left and the most typically resistant to the right. 

Nodule | Diffuse | Macule $\|$ Residual $\left|\begin{array}{c}\text { Simple or } \\ \text { Pale Flat } \\ \text { Macule }\end{array}\right|$ Tuberculoid $\mid \begin{gathered}\text { Major } \\ \text { Tuberculoid }\end{gathered}$ Under lepromatous we have three forms of lesions-nodular, diffuse and mascular; under neural we have major and minor tuberculoid and the simple macule; while in the centre are the residuals of either category. It is generally the two forms on either side of the " residual," namely the lepromatous macule and the simple or pale flat macule, that are difficult to distinguish from each other; but it is not uncommon to confuse even the nodular and the major tuberculoid. The following table gives the main clinical and bacteriological distinctions between the two groups.

\section{Lepromatous.}

I. Many bacilli especially in thickened and nodular lesions. A fair number of bacilli even in the less conspicuous macules.

2. I.esions tend to be symmetrical.

3. Macules are flat and smooth and tend to merge at the margin with the surrounding skin. They have no ringed margin.

4. Macules are numerous and closely placed; they soon coalesce leaving a mottled appearance.

5. Changes of sensation are slight and difficult to elicit; no thickening or tenderness of supplying nerve branches or anidrosis.

6. The lepromin test is negative.

\section{Neural.}

s. No bacilli found as a rule except in the reactionary phase of the major tuberculoid; in these the number of bacilli is small in comparison with the degree of swelling.

2. Lesions tend as a rule to be asymmetrical.

3. Macules (leprides) are raised above the surrounding skin both visibly and palpably. As they increase in size they have a tendency to become flat in the centre and form a ringed margin, the width of which varies greatly in different cases.

4. Macules are, as a rule, few and far apart and may reach a large size.

5. Changes in sensation are more marked as a rule, and there may be thickening and tenderness of supplying nerve branches and anidrosis.

6. The lepromin test is usually positive.

In lepromatous cases in proportion to the number of bacilli there is very little thickening of the skin. Diffuse lesions covering almost the whole body may be almost unnoticeable, especially in dark skins, and yet show many bacilli.

The major tuberculoid is the most conspicuous lesion of the neural type. When activated it is red and swollen and often shows desquamation or scaliness. In severe cases there may be sloughing of the epithelium and ulceration. In such cases a fair number of bacilli may be found, but there is a tendency to spontaneous resolution, the bacilli disappearing and the lesions healing with a greater or less amount of scar formation. 
The minor tuberculoid is similar but less conspicuous.

The simple macule is still less conspicuous. It may spread widely with a fine thin red margin, like a grassland fire in Africa when the grass is short, and a thin line of fire circles the dead, burnt-out area. This type may spread over the face, scarcely being noticed and leaving behind in its scar anaesthesia of the skin, which may be followed by paresis of the underlying facial muscles; this is a common cause of paresis of the eyelids and lips, and of the

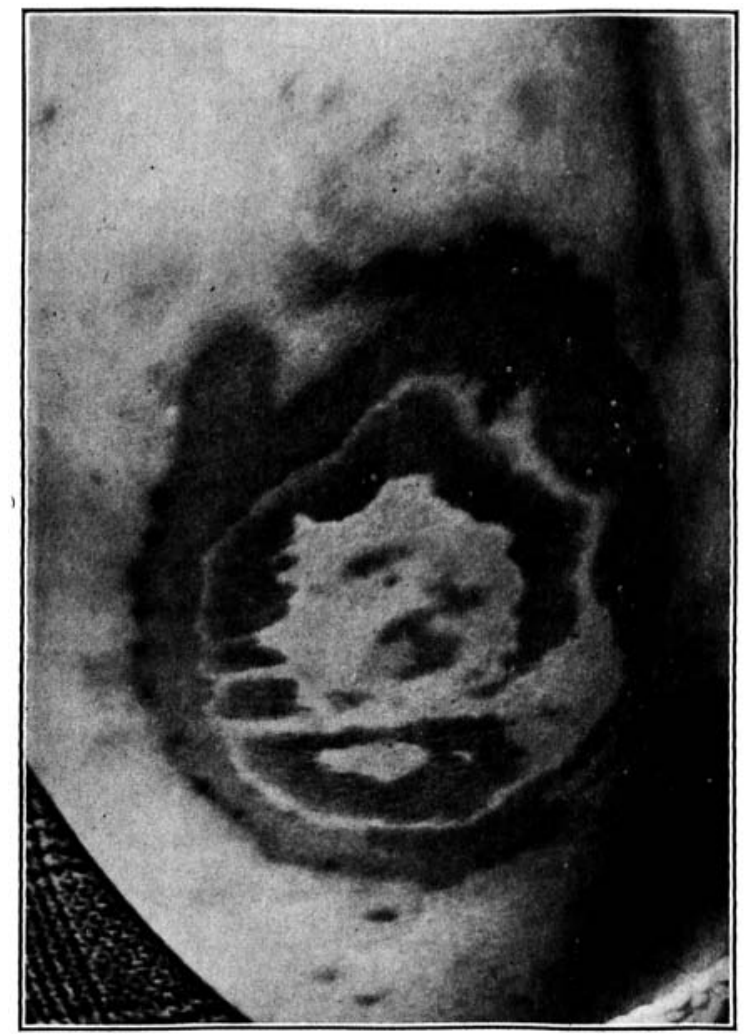

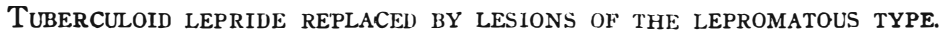

mask-like face, though these may also be caused by the other subtypes of neural lesions.

Residual lesions are of two kinds. (I) In both lepromatous and neural leprosy there are sensory and trophic lesions of the extrem ties and especially of the hands and feet.

(2) The residual lesions of the skin in the lepromatous type are conspicuous in proportion to the thickness and fibrous tissue formation of the previous lesion. The crushed parchment appearance is common as severe lepromatous lesions resolve. 
The major tuberculoid, if deep and inflamed, leaves a conspicuous scar like that of a burn. When less severe, this and the minor tuberculoid may resolve into a pale white macule, similar to the simple macule described above, but without the thin red margin.

We have mentioned above the threshold between lepromatous and neural leprosy resulting, as a rule, in a clear distinction between the lesions characteristic of each type. Apparently this threshold varies in height in different communities. In some communities the threshold is high, lepromatous and neural lesions contrast strongly, and the one form seldom passes into the other. In other communities, especially where climatic and other general conditions are unfavourable and depressing, the threshold may be low, the distinction between the lesions of lepromatous and neural cases may be difficult to discern in a larger proportion of cases, and the neural type may frequently give place to the more severe form. Relics of old tuberculoid lesions may be found in cases which have become distinctly lepromatous; or reference to previous records may show that this change has taken place. In the photo attached a lepromatous lesion has developed in the flattened centre of a residual tuberculoid lepride, and numerous lepromatous macules are seen all over the previously unaffected skin. 